\title{
Anti-influenza virus effects of both live and non-live Lactobacillus acidophilus L-92 accompanied by the activation of innate immunity
}

\author{
Hiroaki Goto ${ }^{1}$, Atsuhiro Sagitani ${ }^{1}$, Nobuhisa Ashida ${ }^{2}$, Shinji Kato ${ }^{1}$, Tatsuhiko Hirota ${ }^{1}$, \\ Tadashi Shinoda ${ }^{3}$ and Naoyuki Yamamoto ${ }^{3 *}$ \\ ${ }^{1}$ Microbiology and Fermentation Laboratory, Calpis Company Limited, 11-10, 5-Chome, Fuchinobe, \\ Sagamihara-shi, Chuo-ku, Kanagawa 252-0206, Japan \\ ${ }^{2}$ Feed Division, Calpis Company Limited, 11-10, 5-Chome, Fuchinobe, Sagamihara-shi, Chuo-ku, \\ Kanagawa 252-0206, Japan \\ ${ }^{3}$ Research and Development Planning Department, Calpis Company Limited, 11-10, 5-Chome, Fuchinobe, \\ Sagamihara-shi, Chuo-ku, Kanagawa 252-0206, Japan
}

(Submitted 2 October 2012 - Final revision received 12 March 2013 - Accepted 12 March 2013 - First published online 18 April 2013)

\begin{abstract}
The antiviral effects of both a live and non-live Lactobacillus acidophilus strain L-92 (L-92) were investigated by oral administration $(10 \mathrm{mg} /$ mouse per d) daily for $21 \mathrm{~d}$ in a mouse model infected intranasally with influenza virus (H1N1). Virus titres in the lung of mice administered either live or non-live L-92 cells daily for $15 \mathrm{~d}$ were repressed $6 \mathrm{~d}$ after virus infection compared with the control group. Natural killer (NK) activity in the orally administered non-live L-92 group was higher compared with that of the control group before virus infection and on day 6. In contrast, NK activity in the live L-92 group compared with the control group was not significantly changed on both days, but was significantly higher on day 1. In contrast, live L-92 showed a greater repression of virus proliferation compared with non-live L-92, 6 d after the infection. Live L-92 decreased the number of neutrophils in the lung and suppressed lung weight, leading to the consequent deterioration of consolidation scores of the lung. These results indicated that pretreatment of live or non-live L-92 cells had protective effects against influenza virus infection. Among the measured cytokines and chemokines, eotaxin, macrophage colony-stimulating factor, IL-1 $\beta$, RANTES (regulated on activation, normal T cell expressed and secreted) and interferon- $\alpha$ were significantly increased in the lung: IL-17 was significantly increased in Peyer's patch of the live L-92 group compared with the control group. A mechanistic study suggested that the enhancement of NK activity in the lung caused by stimulating various antiviral cytokines and chemokines after the oral administration of L-92 cells might be important in protecting against virus infection.
\end{abstract}

Key words: Lactobacillus acidophilus L-92: Innate immunity: Antiviral effects: Natural killer activity: Cytokine profiles

Many probiotic lactic acid bacteria and Bifidobacterium with health benefits to humans, such as improvement of the intestinal environment and allergy symptoms, preventive effects on cancer and lowering of serum cholesterol levels, have been reviewed ${ }^{(1-3)}$. Recent studies have revealed that intranasal administration of Lactobacillus species might be effective in protecting against virus infection and decreasing the relevant inflammation ${ }^{(4,5)}$. Activation of host innate immunity and/or adaptive immunity has been suggested to be important for the protective effect against virus infection ${ }^{(4,5)}$. An orally administered Lactobacillus acidophilus strain L-92 (L-92) has an anti-allergy effect in a mouse model: it has been shown to modulate a T helper (Th) 2-skewed Th1/Th2 immunobalance by increasing the production of IL-12, known as a Th1-type cytokine, and reducing the production of IL-4, known as a Th2-type cytokine, in a mouse model ${ }^{(6)}$. The anti-allergy effects of the oral administration of L-92 have been proven in several previous human trials, including pollen $^{(7)}$ and perennial allergies ${ }^{(8)}$, and atopic dermatitis ${ }^{(9)}$. However, immunostimulating effects, such as the antiviral and anti-pathogenic bacterial effects of L-92, have not yet been elucidated.

The recent outbreak of pandemic H1N1 influenza has been a big social problem. Influenza virus infection to the host respiratory tract mucosa leads to high mortality, and there have been many influenza pandemics, such as the 1918

Abbreviations: IFN, interferon; L-92, Lactobacillus acidophilus strain L-92; M-CSF, macrophage colony-stimulating factor; MIP, macrophage inflammatory protein; PFU, plaque-forming units; PP, Peyer's patch; RANTES, regulated on activation, normal T cell expressed and secreted; Slp, surface layer protein; Th, T helper.

*Corresponding author: Dr N. Yamamoto, fax +81 42769 7810, email naoyuki.yamamoto@calpis.co.jp 
influenza pandemic ${ }^{(10-15)}$. Excessive production of inflammatory cytokines in the lung (cytokine storm) has often been observed in influenza infection, and this causes a severe clinical condition due to an inflammation in the lung ${ }^{(16,17)}$. At present, the use of drugs is the best approach for treatment against virus infection, but there is a risk of the emergence of drug-resistant bacteria due to repeated drug use ${ }^{(18)}$ or that a drug may not be sufficiently effective if they are not taken within about $48 \mathrm{~h}$ of infection ${ }^{(19)}$. Humans have encountered many pandemics caused by new types of influenza virus and tens of thousands of people have died, even though there are many antiviral drugs ${ }^{(13,14,20)}$. We are investigating alternative treatments against virus infection. Many challenging studies have been reported in mice using various probiotic strains to investigate the protective effects against influenza virus infections ${ }^{(21-23)}$. However, these probiotic effects are thought to be species and strain dependent ${ }^{(5)}$. The anti-allergy effect of L-92 is thought to be linked to certain characteristic features in the cell-wall components ${ }^{(24,25)}$. In the present study, we report for the first time the potency of the probiotic L-92 against influenza virus infections. Changes in various cytokines and chemokines were analysed to understand specific L-92 defence mechanisms against virus infection using a mouse model. In addition, the antiviral effects of both live and non-live L-92 cells were compared.

\section{Materials and methods}

\section{Mice}

Female BALB/c mice ( 4 weeks old) were purchased from Japan SLC and allowed to acclimatise for 1 week. Mice were housed in an air-conditioned animal room at $21-27^{\circ} \mathrm{C}$ and $40-80 \%$ humidity under a $12 \mathrm{~h}$ light $-12 \mathrm{~h}$ dark cycle. The experiments were performed in accordance with the Guidelines for Proper Conduct of Animal Experiments (Science Council of Japan, 2006). All experimental procedures were approved by the Calpis Animal Ethical Committee.

\section{Preparation of a live and non-live Lactobacillus} acidophilus strain L-92

L-92 isolated from a healthy Japanese volunteer in our stock culture collection was cultured in a medium consisting of yeast extract (Organotechnie) and casein hydrolysate for $21 \mathrm{~h}$ at $37^{\circ} \mathrm{C}$. The cell culture was centrifuged at $7000 \mathrm{rpm}$ for $15 \mathrm{~min}$ and the pellet cells were washed with sterile PBS. Then, the cells washed with PBS were resuspended in PBS at a concentration of $100 \mathrm{mg}$ wet cells $/ \mathrm{ml}$ (approximately $4 \times 10^{10}$ cells $/ \mathrm{ml}$ : colony-forming units $/ \mathrm{ml}$ ). The number of viable cells was monitored by counting the colonies on the de Man, Rogosa and Sharpe agar plate after the fermentation of the diluted suspension cells for $72 \mathrm{~h}$ at $37^{\circ} \mathrm{C}$. For the preparation of non-live L-92 cells, the washed cells, prepared by the aforementioned method, were heat-killed until the temperature reached $85^{\circ} \mathrm{C}$, then completed freeze-dry treatment and resuspended in PBS at a concentration of $33 \mathrm{mg}$ dried cells $/ \mathrm{ml}$.

\section{Experimental design}

Mice were fed FR-2 (Funabashi Farms) and water was available at all times during the experimental period. As shown in Fig. 1, two sets of experiments were carried out: non-live L-92 was used in Expt I and live L-92 was used in Expt II. In both experiments, L-92 suspension, as prepared above, was administered (Expt I: doses of $300 \mu$ l of non-live L-92 suspension/d, corresponding to $10 \mathrm{mg}$ dry cells/mouse per $\mathrm{d}$ for both experiments; Expt II: doses of $300 \mu \mathrm{l}$ of live L-92 suspension/d) to mice by an oral zonde daily for $21 \mathrm{~d}$. Mice in the control groups received doses of $300 \mu$ l of saline instead of L-92 suspension. The 1st day of sample administration (L-92 or saline) was defined as day -15 , and mice were infected with the influenza virus on day 0. In Expt I, fifty mice were divided into two groups: group I in which NK assays were performed on day 0 before the virus infection; group III in which virus titre, NK assays and Ig assays were performed on day 6, as illustrated in Fig. 1. In Expt II, fiftyfour mice were divided into three groups: group I in which NK assays were performed on day 0 before the virus infection; group II in which virus titre and NK assays were performed on day 1; group III in which virus titre, NK assays, cytokine/ chemokine assays, Ig assays and macroscopic inspections were performed on day 6 . On day 0 , mice in groups II and III were inoculated with the influenza virus. Virus titre analysis of part of the left lung lobe and NK activity measurements from part of the right lung lobe was performed on day 6 in Expt I, and on days 1 and 6 in Expt II (Fig. 1). The remaining left and right lobes were mixed and used for Ig assays on day 6 in Expt I and Expt II, and also cytokine/chemokine analysis on day 6 in Expt II.

\section{Influenza virus and infection}

Influenza $\mathrm{A} / \mathrm{PR} / 8 / 34$ (H1N1) was prepared by Japan Biological Science, Inc., as described previously ${ }^{(21)}$. Mice were inoculated intranasally in the nasal cavity with a $50 \mu \mathrm{l}$ drop of influenza virus at $5 \times 10^{5}$ plaque-forming units (PFU)/ mouse using a micropipette (Eppendorf Company, Limited).

\section{General symptom score}

General symptom scores were calculated by averaging four different health condition scores: eyelid, fur appearance, behaviour and others such as breath and body temperature, as listed in Table 1. Mice were visually inspected for these health conditions, every morning for $21 \mathrm{~d}$, before sample administration, according to the standard operating procedures of Japan Biological Science, Inc. Points were given for each health condition, ranging from 1 (good) to 5 (bad).

\section{Virus titre}

Virus titres in the lung were counted according to the method described previously ${ }^{(21)}$. In short, on day 1 or 6 , a sliced left lung was homogenised with protease inhibitor cocktail (Sigma-Aldrich), and inoculated into MDCK (Madin-Darby 


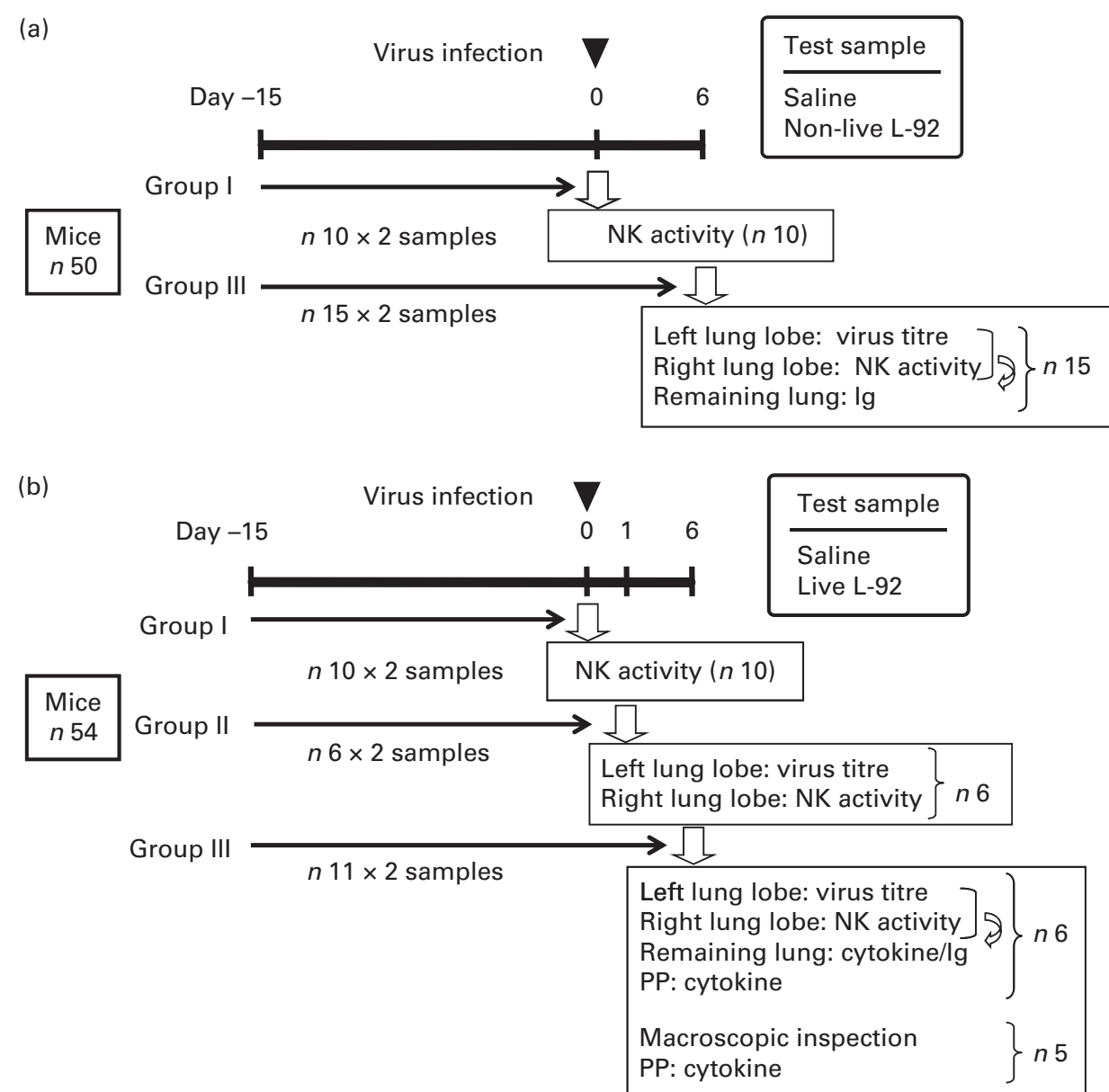

Fig. 1. Experimental design and schedules conducted separately for non-live ((a) Expt I) and live ((b) Expt II) Lactobacillus acidophilus strain L-92 (L-92) cells. L-92 or saline was orally administered daily for the whole study period (days -15 to 6 ). Mice were intranasally infected by the influenza virus on day 0 ( $\mathbf{v}$ ) and some of the mice in the group were used for each analysis $(\Omega)$. Details of the analytical points are listed in the text. NK, natural killer; PP, Peyer's patch.

canine kidney) cells $\left(10^{6}\right.$ cells $\left./ \mathrm{ml}\right)$. Agar medium $(1.5 \mathrm{ml})$ was then added and MDCK cells were incubated for $2 \mathrm{~d}$ at $37^{\circ} \mathrm{C}$. The number of virus plaques was then counted as PFU.

\section{Lung weight and macroscopic evaluation}

Mice were killed and the thorax opened. Lungs were weighed and scored macroscopically, ranging from 1 (no consolidation) to 5 (consolidation through the left or right pulmonary lobe). Scores were averaged if two scores were applied. The consolidation score was calculated by adding the left and right lobe scores, ranging from 2 to 10 .

\section{Number of neutrophils in lung slides}

The left lung was stained using the Giemsa method to count the number of neutrophils. Thereafter, five sites were counted for each lung and the number of cells at each site was averaged.

\section{Natural killer activity in the lung}

NK activity in the lung was evaluated according to a method described previously ${ }^{(21)}$. In short, NK activity was determined using a ${ }^{51} \mathrm{Cr}$ release assay that employed ${ }^{51} \mathrm{Cr}$-labelled YAC-1 cells as target cells. On day 0 before the virus infection, days

Table 1. General symptom scores for the evaluation of health condition of mice

\begin{tabular}{|c|c|c|c|c|}
\hline Score & Eyelid & Fur appearance & Behaviour & Others \\
\hline 5 & Fatal & Fatal & Fatal & Fatal \\
\hline 4 & Blepharosynechia & Dull fur & No reaction when touched & $\begin{array}{l}\text { Respiratory failure/skinny/obvious } \\
\text { decrease of body temperature }\end{array}$ \\
\hline 3 & Loss of eyelid reflex & Piloerection/lose lustre & Loss of reaction when touched & Obvious respiratory irregularity/skinny \\
\hline 2 & $\begin{array}{l}\text { Eyelid closure } \\
\text { (open when touched) }\end{array}$ & Slight piloerection & React when touched & Increased or decreased breathing rate \\
\hline 1 & Normal & Normal & Normal & Normal \\
\hline
\end{tabular}


1 and 6, right lung cells were incubated with radiolabelled $\left({ }^{51} \mathrm{Cr}\right)$ YAC-1 cells. A total of $2 \times 10^{5}$ lung cells were incubated with $1 \times 10^{4}$ target cells at an effector:target ratio of $20: 1$. NK activity was calculated by measuring the radioactivity released from YAC-1 cells according to the following formula:

$$
\begin{aligned}
\text { Cytotoxicity }(\%)= & 100 \times(\text { experimental count } \\
& - \text { spontaneous count }) /(\text { total count } \\
& - \text { spontaneous count }) .
\end{aligned}
$$

\section{Cytokines and chemokines in the lung and Peyer's patch}

The following thirty-four different cytokines and chemokines in the lung and Peyer's patch (PP) extracts prepared from mice on day 6 in Expt I were measured using Multiplex based on an immunological method, according to the manufacturer's instructions (Millipore or Affymetrix): eotaxin; granulocyte colony-stimulating factor; granulocyte macrophage colony-stimulating factor; interferon (IFN)- $\alpha$; IFN- $\beta$; IFN- $\gamma ;$ IL-10; IL-12 (p40); IL-12 (p70); IL-13; IL-15; IL-17; IL-1 $\alpha$; IL-1 $\beta$; IL-2; IL-3; IL-4; IL-5; IL-6; IL-7; IL-9; inducible protein-10; keratinocyte-derived chemokine (KC); leukaemia inhibitory factor; lipopolysaccharide-induced CXC chemokine (LIX); macrophage colony-stimulating factor (M-CSF); monocyte chemoattractant protein-1; monokine induced by IFN- $\gamma$; macrophage inflammatory protein (MIP)- $1 \alpha$; MIP-1 $\beta$; MIP-2; RANTES (regulated on activation, normal $\mathrm{T}$ cell expressed and secreted); TNF- $\alpha$; vascular endothelial growth factor.

\section{Virus-specific IgA and IgG}

MDCK cells seeded on a ninety-six-well plate were infected by PR8 influenza and cultured for $2 \mathrm{~d}$ at $37^{\circ} \mathrm{C}$, according to the method described by Liu et $a l^{(26)}$ with some modification. Then, the influenza-infected cells were immobilised with methanol. The plate prepared above was washed twice with PBS before $100 \mu \mathrm{l}$ of lung extracts from mice were added to each well and incubated for $30 \mathrm{~min}$ at room temperature. Then, a secondary antibody (goat anti-mouse IgA or goat anti-mouse IgG (both peroxidase labelled); Bethyl Laboratories) was added to each well of the plate after being washed with PBS. After the incubation of the plate for $3 \mathrm{~h}$ at room temperature, each $100 \mu$ l of PBS containing $1 \%(\mathrm{w} / \mathrm{v})$ $o$-phenylenediamine dihydrochloride, $4.3 \%(\mathrm{w} / \mathrm{v})$ and $3 \%$ $(\mathrm{v} / \mathrm{v}) \mathrm{H}_{2} \mathrm{O}_{2}$ was added to each well of the washed plate, and incubated for $30 \mathrm{~min}$ at room temperature. After the addition of $100 \mu \mathrm{l}$ of $10 \%(\mathrm{v} / \mathrm{v}) \mathrm{H}_{2} \mathrm{SO}_{4}$ to each well to stop the reaction, optical density at $492 \mathrm{~nm}$ was measured to evaluate optical density. The total amount of Ig (IgA and IgG) was quantified, according to the method described previously ${ }^{(26)}$.

\section{Statistical analyses}

Data are presented as means with their standard errors. Statistical analyses were calculated using Student's $t$ test, two-way ANOVA or the Mann-Whitney test. Differences were considered significant at $P<0.05$ or less.

\section{Results}

The antiviral effects of live and non-live L-92 were investigated in two sets of mouse model studies (Expt I and II), as illustrated in Fig. 1. There was no significant difference in virus titres on day 6 after the infection between the control groups in Expt I (38688 (sE 3877) PFU) and in Expt II (19330 (SE 10250) PFU). Throughout the test period, there was no significant difference in body weight or severe fatal damage (data not shown). General symptom scores were high in the control groups of both experiments throughout the test period, while the non-live group tended to have lower symptom scores compared with the control group $(P<0 \cdot 1)$, but not the live group $(P=0 \cdot 14)$ (Fig. 2). In Expt I using non-live L-92 cells, virus titres in the lung were significantly $(P<0.05)$ lowered in the L-92 group $\left(1.85 \times 10^{4}\right.$ $\left(\mathrm{sE} 0.44 \times 10^{4}\right) \mathrm{PFU}, n$ 15) compared with the control group $\left(3.87 \times 10^{4}\left(\right.\right.$ SE $\left.0.39 \times 10^{4}\right)$ PFU, $n$ 15) on day 6 (Fig. 3(a)). NK activity in the lung was significantly $(P<0 \cdot 001)$ higher in the non-live L-92 group ( 4.70 ( $\operatorname{se} 0.55) \%, n$ 15) compared with the control group (0.90 (SE 0.16)\%, $n$ 15) on day 6 (Fig. 3(b)). Then, in Expt II using live L-92 cells (viability over $70 \%$ ), virus titre and NK activity in the lung were monitored on day 1 to understand the quick host response after the virus infection. As a result, virus titre of the control group on day 1 (300 (sE 300) PFU, $n$ 6) increased dramatically on day 6 in the control group $\left(1.9 \times 10^{4}\left(\right.\right.$ se $\left.1.0 \times 10^{4}\right)$ PFU, $n$ 6), but not in the L-92 group (Fig. 4(a)). NK activity in the lung on day 1
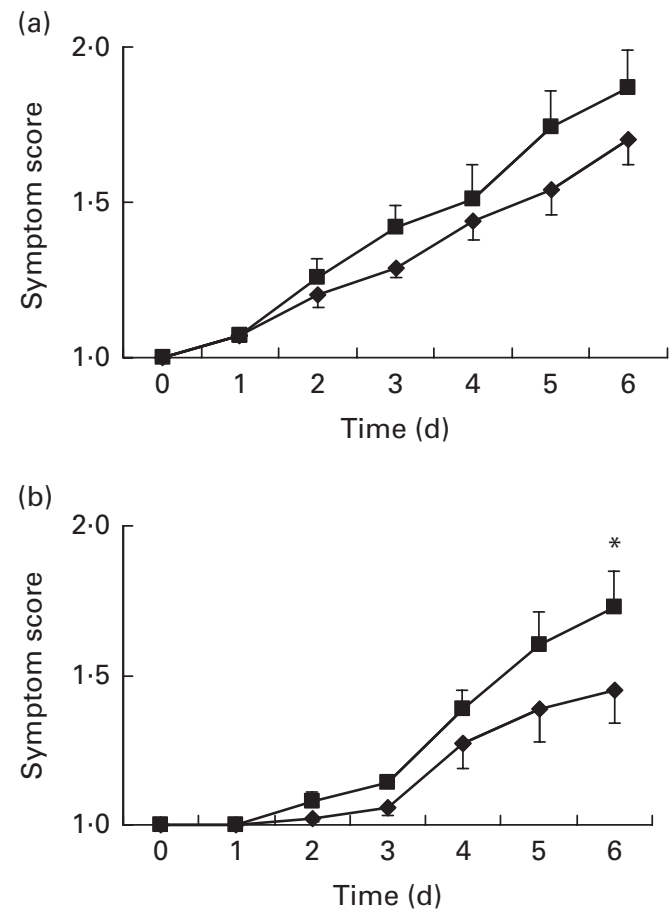

Fig. 2. General symptom scores of mice administered (a) live $(\bullet-)$ or (b) non-live ( $\bullet$ ) Lactobacillus acidophilus strain L-92 (L-92) cells and the saline-administered control (- $)$ group from days 0 to 6 . L-92 or saline was administered daily for the whole study period (days -15 to 6 ). Values are means ((a) $n 11$ and (b) $n$ 15), with their standard errors represented by vertical bars. ${ }^{*}$ Mean values between groups were marginally significantly different $(P<0 \cdot 1$; two-way ANOVA). 


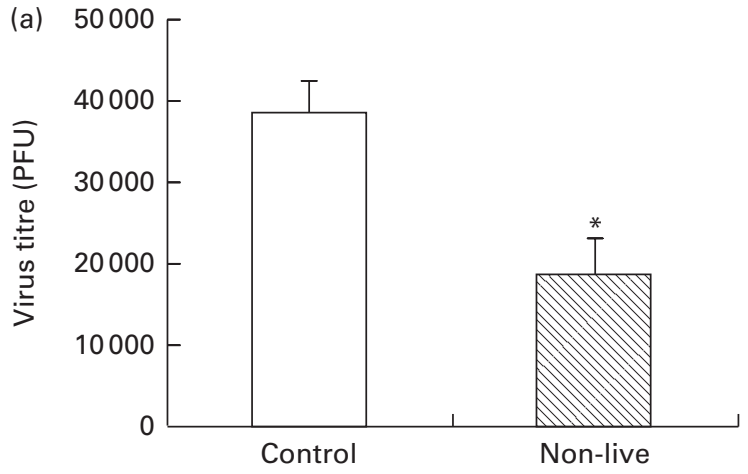

(b)

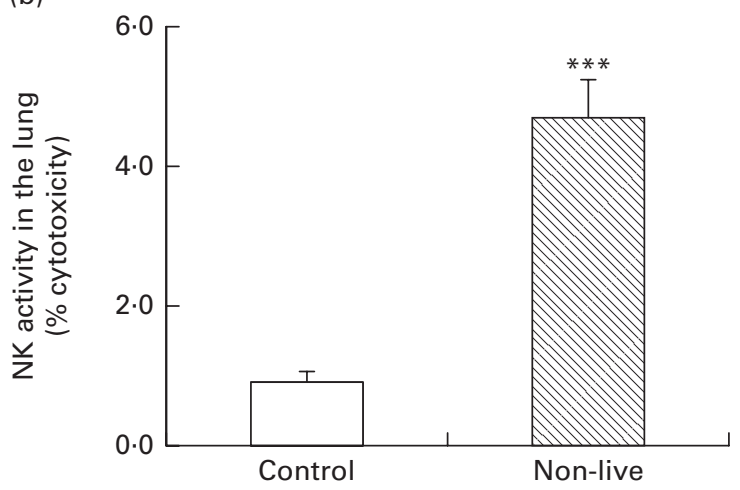

Fig. 3. (a) Virus titres and (b) natural killer (NK) activity in the lung of mice administered non-live Lactobacillus acidophilus strain L-92 (L-92) and the saline-administered control groups on day 6. L-92 or saline was administered daily for the whole study period (day -15 to 6). Values are means ((a) $n 15$ and (b) $n$ 15), with their standard errors represented by vertical bars. Mean value was significantly different compared with the control group: ${ }^{*} P<0.05$, ${ }^{\star \star \star} P<0.001$ (Student's $t$ test). PFU, plaque-forming units.

was significantly $(P<0.05)$ higher in the live L-92 group (3.30 (sE $0 \cdot 16) \%, n$ 6) compared with the control group $(1 \cdot 70$ (SE 0.55)\%, n 6) (Fig. 4(b)). However, interestingly, no differences in NK activities were observed between the two groups on day 6 in Expt II (Fig. 4(b)). These results suggested that mice pre-administered either live or non-live L-92 cells showed protective effects against influenza virus infection and/or proliferation.

There were similar host responses between the live and non-live L-92 groups in virus titres on day 6; however, host responses in NK activities in the lung of both groups were quite different (Figs. 3(b) and 4(b)). Notably, the repression of the virus titre in the live L-92 group compared with the control group was significantly greater on day 6 but not on day 1; however, NK activity was significantly activated on day 1 and not on day 6 (Fig. 4). NK activity in the non-live group was also significantly activated on day 0 before the infection (after $15 \mathrm{~d}$ of L-92 administration) compared with the control group ( $4 \cdot 18$ (sE 0.48), $n$ 10), but was not significantly changed in the live L-92 group (2.68 (SE 0.54), $n$ 10) compared with the control group (2.28 (SE 0.08), $n$ 10) (Fig. 5). These results suggested that live L-92 cells might have a greater (or quicker) effect in the prevention of the influenza virus infection.
Thus, live L-92 cells were selected and used in the subsequent study for a more detailed understanding of the host response after the infection of the influenza virus. After the infection of influenza virus, inflammatory host damage in the lung is usually observed as the main host event, so the consolidation score of the lung for the live L-92-treated group was compared with that for the saline-treated control group. The live L-92 group showed a significant decrease in consolidation scores (3.00 (se 0.35), $n 5, P<0.001)$ compared with the control group (6.00 (SE 0.55), $n$ 5) (Fig. 6(a)). In addition, the number of neutrophils in the lung was significantly $(P<0.05)$ lowered in the L-92 group (46.7 (SE 8.0), $n$ 5) compared with the control group (80.3 (SE 11.1), $n$ 5) (Fig. 6(b)). Lung weight is known to be increased by inflammation; here it was significantly $(P<0.05)$ lowered in the live L-92 group (171.3 (SE 13.7) $\mathrm{mg}, n$ ) compared with the control group (232.7 (sE 26.4) $\mathrm{mg}, n$ ).

To understand the host response, the antiviral effect mechanism and anti-inflammatory events most probably linked to the virus infection: the release of various cytokines and chemokines in the lung and PP was measured in the live L-92 group on day 6 . The thirty-four different cytokines and chemokines were measured, which showed that eotaxin, M-CSF, IL-1 $\beta$, RANTES and IFN- $\alpha$ were significantly increased in the lung of the live L-92 group compared with the control group (Fig. 7(a)). Furthermore, there were significant increases in the production of IL-17, while IFN- $\alpha$ tended to be increased and IL- 4 and IL- 6 tended to be decreased in PP, $6 \mathrm{~d}$ after the virus infection (Fig. 7(b)). As expected from the protective anti-influenza virus effect shown in Figs. 2-4, notable increases in IFN- $\alpha$, which is known to be activated in response to virus infection, were observed in the lung and PP (Fig. 7(a) and (b)). In addition, IFN- $\beta$ was increased in the lung of mice from the L-92 group $(310$ (SE 280) pg/ml, $P=0.33)$, although this was not significant when compared with the control group $(17 \cdot 4 \quad(\mathrm{SE} 3.5) \mathrm{pg} / \mathrm{ml})$. IL-6 was decreased in the lung in the L-92 group $(4 \cdot 4$ (SE 2.3) ng $/ \mathrm{ml}$, $P=0 \cdot 31)$, although this was not significant when compared with the control group (8.9 ( $\mathrm{SE} 3.5) \mathrm{ng} / \mathrm{ml}$ ).

$\operatorname{IgA}$ and $\operatorname{IgG}$ were measured in the lung on day 6 to understand the effect of live and non-live L-92 on adaptive immunity. A small, but significant $(P<0.05)$ decrease in the amount of $\operatorname{IgG}$ was observed in the live L-92 group $(0 \cdot 12$ (SE 0.004), $n$ 6) when compared with the control group $(0.14$ ( $\operatorname{se} 0.005), n 6)$, but not in the non-live L-92 group (0.15 (SE 0.005), $n$ 15) when compared with the control group (0.14 (SE 0.005), $n$ 15). There was no significant change in IgA in the live and non-live L-92 groups compared with the control group (data not shown).

\section{Discussion}

There have been many reports on the protective effects of the probiotic Lactobacillus and Bifidobacterium strains against influenza virus infection in mice ${ }^{(5,21-23,27)}$. These effects seem to be species and strain dependent. Youn et al. ${ }^{(5)}$ reported strain-specific clinical efficacy and differences in the effects among strains used in their clinical study. So far, 

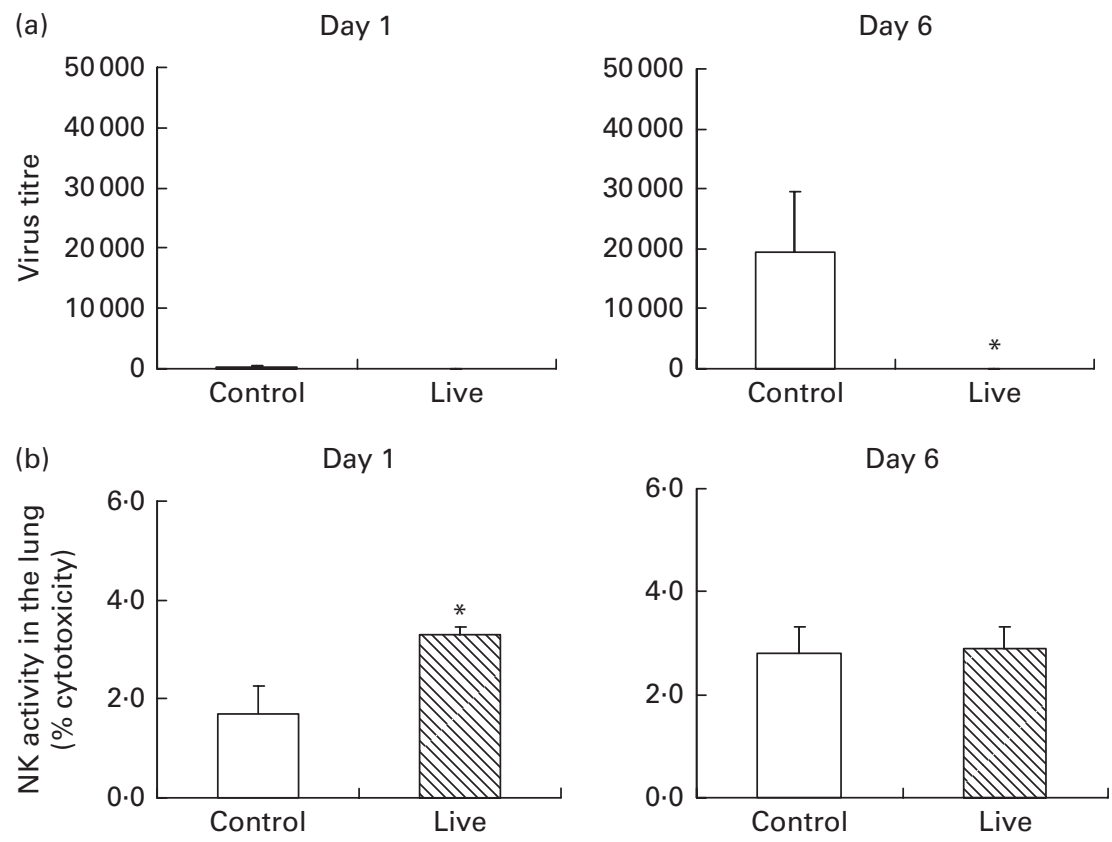

Fig. 4. (a) Virus titres and (b) natural killer (NK) activity in the lung of mice administered live Lactobacillus acidophilus strain L-92 (L-92) and the saline-administered control groups on days 1 and 6. L-92 or saline was administered daily for the whole study period (days -15 to 6$)$. Values are means ((a) $n 6$ and (b) $n 6)$, with their standard errors represented by vertical bars. * Mean value was significantly different compared with the control group $(P<0.05 ;$ Student's $t$ test).

there has been only one report of an antiviral effect of L. acidophilus in a human trial ${ }^{(28)}$, and no detailed mechanism studies or discussions about the mode of action have been published. This is the first report of the protective effects of a $L$. acidophilus strain against influenza virus infection in a mouse model and the first mechanism study considering the effect. This is also the first comparative study using live and non-live L. acidophilus cells analysing the antiviral effects. L-92 is known for the immunomodulatory effect in some allergy symptoms, such as pollen ${ }^{(7)}$ and perennial allergies ${ }^{(8)}$, and atopic dermatitis ${ }^{(9)}$ in humans. The present study also reveals a potential immunomodulatory effect of L-92, not only for anti-allergy effects, but also for an immunostimulation effect, such as protective effects against pathogenic bacteria and virus infection in vivo.

In the present study, both live and non-live L-92 cells showed antiviral effects in separate mouse model experiments. However, host responses after the oral administration of live and non-live cells differed. NK activity before the virus infection was higher in the non-live group compared with the live group; however, virus titres in the lung $6 \mathrm{~d}$ after the infection were more repressed in the live group (more than 99\%) than in the non-live group (55\%) when compared with the control groups. Furthermore, NK activity in the non-live group was stimulated more than 5-fold $6 \mathrm{~d}$ after the virus infection when compared with the control group, but not in the live group. These results suggest that elimination of the virus from the lung, based on NK activity-dependent innate immunity, was higher in the non-live group than in the live group. However, elimination of the virus from the lung in the live group was higher than that in the non-live group. One of the possible explanations would be quick and temporary activation of NK activity by the virus infection on day 1 (Fig. 4(b)) that reaches to following thorough elimination of the virus in the live L-92 group observed on day 6 (Fig. 4(a)), and disappeared NK activation on day 6 (Fig. 4(b)). In contrast, the increase of NK activity in the non-live L-92 group appearing on day 6 may be due to a delayed host response compared with the live group, which may affect on the following elimination of the virus in the lung after day 6 (not tested). Higher preventive effects against influenza virus infection in a live bacteria-administered group compared with a heatkilled bacterial group have been reported in a previous study ${ }^{(5)}$. With respect to the general symptom score, there seemed to be a greater improvement in the non-live group $(P<0.1 v$. control group, $n$ 15) than in the live group $(P=0.14 v$. control group, $n$ 11) throughout the test period (Fig. 2). The possible reason for this difference must be

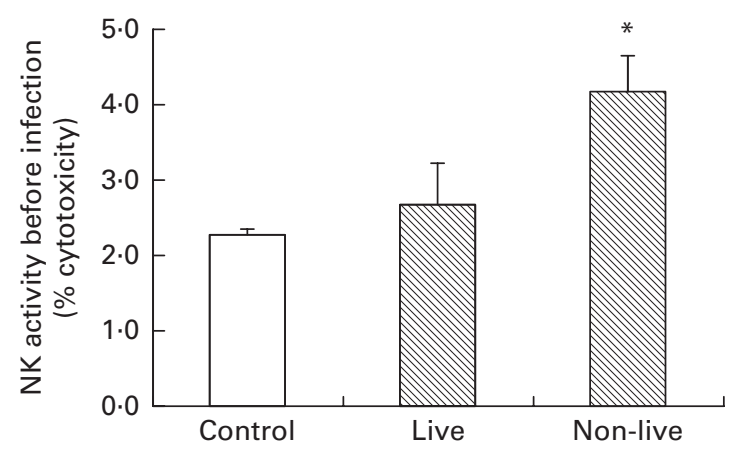

Fig. 5. Natural killer (NK) activity in the lung of mice administered live or nonlive Lactobacillus acidophilus strain L-92 cells or saline on day 0 before the virus infection. Values are means (n 10), with their standard errors represented by vertical bars. ${ }^{*}$ Mean value was significantly different compared with the control group ( $P<0.05$, Student's $t$ test). 

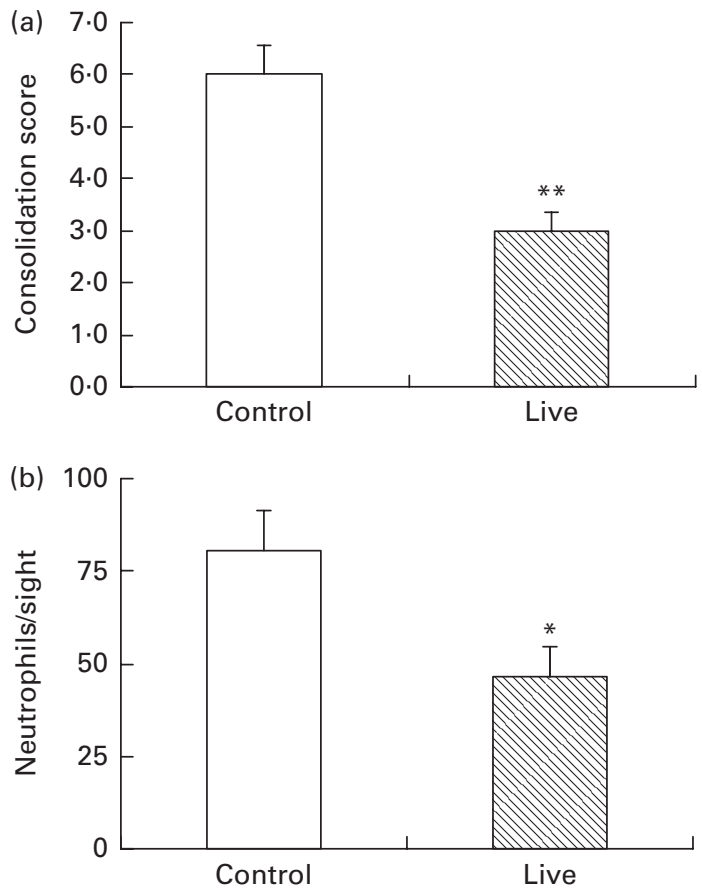

Fig. 6. (a) Consolidation score and (b) the number of neutrophils in the lung of mice administered live Lactobacillus acidophilus strain L-92 (L-92) cells and saline on day 6. L-92 or saline was administered daily for the whole study period (days -15 to 6). Values are means ((a) $n 5$ and (b) $n 5$ ), with their standard errors represented by vertical bars. Mean value was significantly different compared with the control group: ${ }^{*} P<0.05,{ }^{\star *} P<0.01$ (Student's $t$ test).

because of the smaller number of mice used in the experiments for the live group. The statistical calculation suggested that the general symptom score in the live group would be significantly lower than that of the control group $(P<0.05)$ if the number of mice was increased to 15 . However, a detailed comparative study on the mechanism of host responses against virus infection and immune response including NK activity and some cytokines between live and non-live, and also different administration routes, should be addressed in the future.

In the present study, among the measured cytokines and chemokines, eotaxin, M-CSF, IL-1 $\beta$, RANTES and IFN- $\alpha$ were significantly increased in the lung of the live L-92 group compared with those in the control group. In a previous study, influenza infection of $\mathrm{C} 57 \mathrm{BL} / 6 \mathrm{~J}$ mice induced chemokine gene expression for monocyte chemoattractants, monocyte chemotactic protein-1, MIP-1 $\alpha, \quad M I P-1 \beta, \quad$ RANTES and IFN-inducible protein $10^{(29)}$. RANTES was considered to be important for virus infection because knockout $(-/-)$ mice did not demonstrate increased susceptibility to influenza infection $^{(29)}$. M-CSF enhancement has been suggested to be important for early host resistance to influenza virus ${ }^{(30)}$. A potent eosinophil chemoattractant, eotaxin, well known to be induced after influenza virus infection in nasal epithelial cells ${ }^{(31)}$, was significantly induced in the present study. At $6 \mathrm{~d}$ after the virus infection, there was a significant increase in the production of IL-17, IFN- $\alpha$ tended to be increased and IL- 4 and IL-6 were decreased in PP. Influenza virus infection was accompanied by IL-1 $\beta$ and IFN- $\alpha$ production in the bronchoalveolar lavage of mice ${ }^{(32)}$. The production of antiviral cytokines, such as IFN- $\alpha$ and $-\beta$ which have been reported to induce $\mathrm{NK}$ activity ${ }^{(33-35)}$, was measured $6 \mathrm{~d}$ after the virus infection in the present experiment. The antiviral cytokine IFN- $\alpha$ was significantly increased in both lung and PP compared with the control group. IFN- $\beta$ in the L-92 group was present at a higher value than that in the control group $(P=0.33)$. In addition, other cytokines (IL-2 and IL-15), which are also known to induce NK activity, were not significantly different, but their average values were both higher in the L-92 group compared with the control group. The enhancement of NK activity in the lung may be caused by stimulating various antiviral cytokines after the oral administration of L-92 cells. An evaluation of various cytokines and chemokines before the virus infection would be of interest in considering the host response against probiotic challenge with or without virus infection.

Mohamadzadeh et $a l .{ }^{(36)}$ reported that surface components of a Lactobacillus strain were the most important factor in the intake of active cells via $M$ cells in the gastrointestinal tract and the activation of dendritic cells to induce a host immunomodulation effect. Perdigón et al. ${ }^{(37)}$ also reported that several kinds of surface components in lactic acid
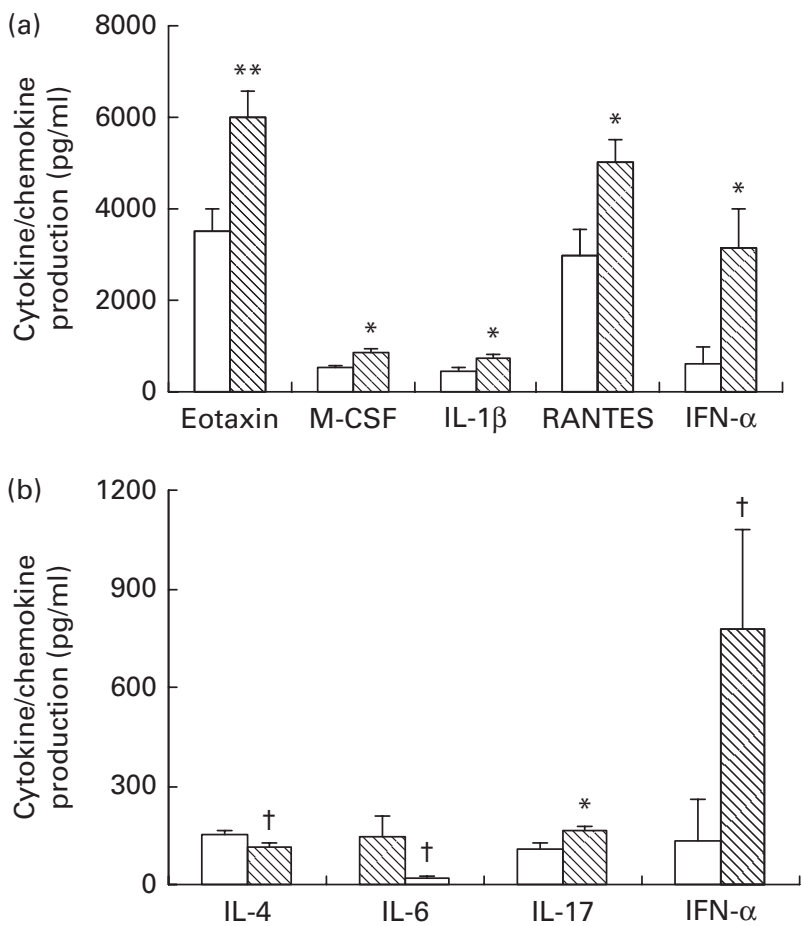

Fig. 7. Cytokine and chemokine production in (a) the lung and (b) Peyer's patch (PP) on day 6 of mice administered live Lactobacillus acidophilus strain L-92 cells or saline daily for the whole study period (day -15 to 6 ). The production of the thirty-four different cytokines and chemokines was evaluated. Values are means ((a) $n 6$ and (b) $n$ 11), with their standard errors represented by vertical bars. Mean value was significantly different compared with the control group: ${ }^{\star} P<0.05,{ }^{*} P<0.01$ (Student's $t$ test). † Mean value was marginally significantly different compared with the control group $(P<0.1$; Student's $t$ test). M-CSF, macrophage colony-stimulating factor; RANTES, regulated on activation, normal $\mathrm{T}$ cell expressed and secreted; IFN, interferon. $\square$, Control; $₫$, live. 
bacteria, such as surface layer protein (Slp), lipoteichoic acid and peptideglycan, are involved in the interaction with host cells. In addition, the importance of SlpA in L. acidophilus NCFM in association of the cell with immature dendritic cells, which is essential for immunomodulatory action, has been suggested ${ }^{(38)}$. A recent study has reported on the potential of L. acidophilus ATCC 4365 to inhibit Junin virus entry using Slp on L. acidophilus to bind to dendritic cellspecific intracellular adhesion molecule (ICAM)-3-grabbing nonintegrin that inhibited the entry of the virus ${ }^{(39)}$. An inhibitory effect of Slp on the adhesion and entry of several virus families has also been suggested ${ }^{(39)}$. In our previous study, probiotic L-92-enriched GroEL and GroES have been considered to have the potential to affect immunomodulation in mouse splenocytes ${ }^{(24)}$. Moreover, a comparison of L. acidophilus strains showed that L-92 expressed higher amounts of cell-wall Slp and produced greater amounts of the Th1-type cytokine IL-12 after incubation with splenocytes ${ }^{(25)}$. These reports have suggested that L-92 has the potential to protect against virus infection via Slp binding to DC-specific ICAM-3-grabbing nonintegrin on epithelial cells. There may also be structural changes in the cell wall and membrane components of live and non-live cells after heat treatment, leading to changes in host responses in the gastrointestinal immune system. To further understand the antiviral effect of L-92 cells, clarification of the key active components and the differences between live and non-live cells should be addressed.

In the present study, adaptive immunity was not thought to be involved because there were no significant increases in IgA and $\operatorname{IgG}$ in the live L-92 group. IgA in the lung $6 \mathrm{~d}$ after the infection in the L-92 group was not significantly changed compared with the control group. IgG in the live L-92 group was slightly, but significantly, lower compared with the control group. The lower adaptive immune responses are thought to be due to the delayed production of $\operatorname{IgG}$ and IgA after the virus infection (about 1 week after the infection). Generally, lactobacilli have been reported to induce adaptive immunity by increasing $\operatorname{Ig}^{(40)}$; however, in the present study, they were not increased in the live L-92 group. We suggest that pretreatment of L-92 cells activates innate immunity so effectively that it causes immediate elimination of virus infection, or the mode of L-92 action differs from other lactobacilli.

A number of studies in animals have demonstrated the potential of using probiotics as immune adjuvants. Lactobacillus species and other probiotics stimulate both the cellular and innate immune systems ${ }^{(41)}$. Clinical trials have also supported the potential of Lactobacillus in the prevention of influenza infection $^{(22,27)}$. In a clinical trial on thirty-nine subjects, Lactobacillus GG showed a promising immunoadjuvant effect against the vaccine $\mathrm{H} 1 \mathrm{~N} 1$ and B strain ${ }^{(42)}$. In contrast, a large clinical study has shown no significant protective effects against respiratory symptoms when volunteers received daily doses of Lactobacillus casei Shirota for $176 \mathrm{~d}^{(43)}$. It may be necessary to conduct a clinical study using the L-92 strain to investigate the potential protective effects against virus infection.

In conclusion, a protective effect against the nasal infection of influenza virus in both live and non-live L-92 cells showed potential in reducing the risk of the virus infection, and may be extended to other types of virus. The antiviral effect was considered to be caused by the early activation of NK activity in the lung caused by stimulating various antiviral cytokines and chemokines during the infection period.

\section{Acknowledgements}

The authors thank A. Morikawa and H. Tsunoda (Nihon Bioresearch, Inc.) for technical assistance throughout the two animal studies. H. G. and N. Y. analysed the data, wrote and revised the paper; A. S., N. A., T. H. and T. S. conceived the design, performed the experiments and acquired the data; S. K. performed the experiments. There was no funding for the present study. The authors have no conflicts of interest to declare.

\section{References}

1. Gill HS \& Guarner F (2004) Probiotics and human health: a clinical perspective. Postgrad Med J 80, 516-526.

2. Michail S (2009) The role of probiotics in allergic diseases Allergy Asthma Clin Immunol 5, 1-7.

3. Liong MT (2008) Roles of probiotics and prebiotics in colon cancer prevention: postulated mechanisms and in-vivo evidence. Int J Mol Sci 9, 854-863.

4. Gabryszewski SJ, Bachar O, Dyer KD, et al. (2011) Lactobacillus-mediated priming of the respiratory mucosa protects against lethal pneumovirus infection. I Immunol 186, 1151-1161.

5. Youn HN, Lee DH, Lee YN, et al. (2012) Intranasal administration of live Lactobacillus species facilitates protection against influenza virus infection in mice. Antiviral Res 93, $138-143$.

6. Torii A, Torii S, Fujiwara S, et al. (2007) Lactobacillus acidophilus strain L-92 regulates the production of Th1 cytokine as well as Th2 cytokines. Allergol Int 56, 293-301.

7. Ishida Y, Nakamura F, Kanzato H, et al. (2005) Effect of milk fermented with Lactobacillus acidophilus strain L-92 on symptoms of Japanese cedar pollen allergy: a randomized placebo-controlled trial. Biosci Biotechnol Biochem 69, 1652-1660.

8. Ishida Y, Nakamura F, Kanzato H, et al. (2005) Clinical effects of Lactobacillus acidophilus strain L-92 on perennial allergic rhinitis: a double-blind, placebo-controlled study. J Dairy Sci 88, $527-533$.

9. Torii S, Torii A, Itoh K, et al. (2011) Effects of oral administration of Lactobacillus acidophilus L-92 on the symptoms and serum markers of atopic dermatitis in children. Int Arch Allergy Immunol 154, 236-245.

10. Tamura S \& Kurata T (2004) Defense mechanisms against influenza virus infection in the respiratory tract mucosa. Jpn J Infect Dis 57, 236-247.

11. Tregoning JS \& Schwarze J (2010) Respiratory viral infections in infants: causes, clinical symptoms, virology, and immunology. Clin Microbiol Rev 23, 74-98.

12. Zhang C, Xu Y, Jia L, et al. (2010) A new therapeutic strategy for lung tissue injury induced by influenza with CR2 targeting complement inhibitor. Virol J 7, 30

13. Taubenberger JK \& Morens DM (2006) 1918 Influenza: the mother of all pandemics. Emerg Infect Dis 12, 15-22.

14. Taubenberger JK \& Morens DM (2008) The pathology of influenza virus infections. Annu Rev Pathol 3, 499-522. 
15. Korteweg C \& Gu J (2010) Pandemic influenza A (H1N1) virus infection and avian influenza A (H5N1) virus infection a comparative analysis. Biochem Cell Biol 88, 575-587.

16. Walsh KB, Teijaro JR, Wilker PR, et al. (2011) Suppression of cytokine storm with a sphingosine analog provides protection against pathogenic influenza virus. Proc Natl Acad Sci U S A 108, 12018-12023.

17. Salomon R, Hoffmann E \& Webster RG (2007) Inhibition of the cytokine response does not protect against lethal H5N1 influenza infection. Proc Natl Acad Sci U S A 104, 12479-12481.

18. Regoes RR \& Bonhoeffer S (2006) Emergence of drug resistant influenza virus: population dynamical considerations. Science 312, 389-391.

19. Aokil FY, Macleod MD, Paggiaro P, et al. (2003) Early administration of oral oseltamivir increases the benefits of influenza treatment. J Antimicrobial Chemotherapy 51, $123-129$

20. Fraser C, Donnelly CA, Cauchemez S, et al. (2009) Pandemic potential of a strain of influenza A (H1N1): early findings. Science 324, 1557-1561

21. Kawase M, He F, Kubota A, et al. (2012) Heat-killed Lactobacillus gasseri TMC0356 protects mice against influenza virus infection by stimulating gut and respiratory immune responses. FEMS Immunol Med Microbiol 64 , $280-288$

22. Davidson LE, Fiorino AM, Snydman DR, et al. (2011) Lactobacillus GG as an immune adjuvant for live-attenuated influenza vaccine in health adults: a randomized doubleblind placebo-controlled trial. Eur J Clin Nutr 65, 501-507.

23. Namba K, Hatano M, Yaeshima T, et al. (2010) Effects of Bifidobacterium longum BB536 administration on influenza infection, influenza vaccine antibody titer, and cell-mediated immunity in the elderly. Biosci Biotechnol Biochem $\mathbf{7 4}$, 939-945.

24. Kuwana R \& Yamamoto N (2012) Increases in GroES and GroEL from Lactobacillus acidophilus L-92 in response to a decrease in medium $\mathrm{pH}$, and changes in cytokine release from splenocytes: transcriptome and proteome analyses. J Biosci Bioeng 114, 9-16.

25. Ashida N, Yanagihara S, Shinoda T, et al. (2011) Characterization of adhesion molecule with affinity to $\mathrm{CacO}-2$ cells in Lactobacillus acidophilus by proteome analysis. J Biosci Bioeng 112, 333-337.

26. Liu B, Hossain MJ, Mori I, et al. (2008) Evaluation of a virus derived from MDCK cells infected persistently with influenza A virus as a potential live-attenuated vaccine candidate in the mouse model. J Med Virol 80, 888-894.

27. Oliveres M, Díaz-Ropero PM, Sierra S, et al. (2007) Oral intake of Lactobacillus fermentum CECT5716 enhances the effects of influenza vaccination. Nutrition 23, 254-260.
28. Leyer GJ, Li S, Mubasher ME, et al. (2009) Probiotic effects on cold and influenza-like symptom incidence and duration in children. Pediatrics 124, e172-e179.

29. Wareing $\mathrm{MD}$, Lyon $\mathrm{AB}$, Lu B, et al. (2004) Chemokine expression during the development and resolution of a pulmonary leukocyte response to influenza A virus infection in mice. J Leukoc Biol 76, 886-895.

30. Sever-Chroneos Z, Murthy A, Davis J, et al. (2011) GM-CSF modulates pulmonary resistance to influenza A infection. Antiviral Res 92, 319-328.

31. Kawaguchi M, Kokubu F, Kuga H, et al. (2000) Expression of eotaxin by normal airway epithelial cells after influenza virus A infection. Int Arch Allergy Immunol 122, Suppl. 1, 44-49.

32. Hennet T, Zilterner JH, Frei K, et al. (1992) A kinetic study of immune mediators in the lungs of mice infected with influenza A virus. J Immunol 149, 935-939.

33. Biron CA (2001) Interferons alpha and beta as immune regulators - a new look. Immunity 14, 661-664.

34. Johansson C, Wetzel JD, He J, et al. (2007) Type I interferons produced by hematopoietic cells protect mice against lethal infection by mammalian reovirus. $J$ Exp Med 204, $1349-1358$

35. Durbin JE, Fernandez-Sesma A, Lee CK, et al. (2000) Type I IFN modulates innate and specific antiviral immunity. J Immunol 164, 4220-4228.

36. Mohamadzadeh M, Duong T, Hoover T, et al. (2008) Targeting mucosal dendritic cells with microbial antigens from probiotic lactic acid bacteria. Expert Rev Vaccines 7 , 163-174.

37. Perdigón G, Fuller R \& Raya R (2001) Lactic acid bacteria and their effect on the immune system. Curr Issues Intest Microbiol 2, 27-42.

38. Konstantinov SR, Smidt H, de Vos WM, et al. (2008) S layer protein A of Lactobacillus acidophilus NCFM regulates immature dendritic cell and $\mathrm{T}$ cell functions. Proc Natl Acad Sci U S A 105, 19474-19479.

39. Martínez MG, Acosta MP, Candurra NA, et al. (2012) S-layer proteins of Lactobacillus acidophilus inhibits JUNV infection. Biochem Biophys Res Commun 422, 590-595.

40. Wells JM (2011) Immunomodulatory mechanisms of lactobacilli. Microb Cell Fact 10, Suppl. 1, S17.

41. MacDonald TT \& Bell I (2010) Probiotics and the immune response to vaccines. Proc Nutr Soc 69, 442-446.

42. Harata G, He F, Hiruta N, et al. (2010) Intranasal administration of Lactobacillus rhamnosus GG protects mice from H1N1 influenza virus infection by regulating respiratory immune responses. Lett Appl Microbiol 50, 597-602.

43. Puyenbroeck VK, Hens N, Coenen S, et al. (2012) Efficacy of dairy intake of Lactobacillus casei Shirota on respiratory symptoms and influenza vaccination immune response: a randomized, double-blind, placebo-controlled trial in healthy elderly nursing home residents. Am J Clin Nutr 95 , $1165-1171$. 